\title{
Effectiveness of Waist Circumference in Assessing the Health Risk by Evaluating the Cardio Metabolic Risk Factors
}

\author{
Vinnakota Shiva Prasad ${ }^{1}$, Vinnakota Sindhura ${ }^{2}$, Mallineni Naveen Kumar ${ }^{3}$ \\ ${ }^{1}$ Consultant, Department of Family Medicine, Padmaja Hospital, Vizianagaram, Andhra Pradesh, India. ${ }^{2}$ Medical \\ Officer, SNCU, Department of Paediatrics, King George Hospital, Visakhapatnam, Andhra Pradesh, India. ${ }^{3}$ Civil \\ Assistant Surgeon, Department of Plastic Surgery, Visakha Institute of Medical Sciences, Visakhapatnam, \\ Andhra Pradesh, India.
}

\section{ABSTRACT}

\section{BACKGROUND}

Obesity is an important risk factor for cardio metabolic diseases like Diabetes, Hypertension, Dyslipidaemia and Coronary Heart Disease (CHD). Several organizations like WHO, National Institutes of Health tried to classify Obesity on basis of weight using BMI. Several epidemiological studies revealed direct correlation between BMI and the risk of medical complications and mortality rate. Body fat distribution is also an important risk factor for obesity-related diseases. Excess abdominal fat also known as central or upper body fat is associated with increased risk of cardio metabolic disease. Janssen et al reported that increased visceral fat, as measured by MRI correlated well with Waist circumference (WC) than with body mass index (BMI). Zhu et al using the third National Health and Nutrition Examination Survey data reported that WC is more closely linked to cardiovascular disease risk factors than BMI. We aimed to determine the effectiveness of waist circumference (WC) in assessment of Health Risk by evaluating the cardio metabolic risk factors.

\section{METHODS}

The sample included 238 middle aged factory employees attending the hospital for periodical check-up. This is a cross-sectional hospital-based study. Blood samples are collected in fasting for Triglycerides, LDL, HDL and HbA1c estimation, BP and waist circumference measured. Chi-square is applied to test the goodness of fit to verify the distribution of observed data with assumed theoretical and expected frequencies. Null hypotheses tested with Chi-square.

\section{RESULTS}

$47.8 \%$ subjects of $>100 \mathrm{cms}$ waist circumference group documented $>400 \mathrm{mg} \%$ Triglyceride levels against $21.8 \%$ subjects in $\leq 90 \mathrm{cms}$ waist circumference group. $50 \%$ subjects of $>100 \mathrm{cms}$ waist circumference group documented $>130 \mathrm{mg} \%$ LDL levels against $21.8 \%$ subjects in $\leq 90 \mathrm{cms}$ waist circumference group. $45.7 \%$ subjects of $>100 \mathrm{cms}$ waist circumference group documented $<40 \mathrm{mg} \%$ HDL levels against $19.1 \%$ subjects in $\leq 90 \mathrm{cms}$ waist circumference group. $43.5 \%$ subjects of $>100 \mathrm{cms}$ waist circumference group documented $>6.5 \%$ HbA1c levels against $9.1 \%$ subjects in $\leq 90 \mathrm{cms}$ waist circumference group. Only 10\% subjects had normal BP in the group of $>100 \mathrm{cms}$ waist circumference against $80 \%$ subjects in the low Waist Circumference group ( $<90 \mathrm{cms}$ ). Waist Circumference is well correlated with Triglycerides, LDL, HbA1c and BP readings but inversely correlated with HDL.

\section{CONCLUSIONS}

Increasing levels of Waist circumference correlate with several biomarkers of cardiovascular disease and diabetes.

\section{KEY WORDS}

Waist Circumference, Triglycerides, Low Density Lipoproteins, High Density Lipoproteins, Glycosylated haemoglobin (HbA1c)
Corresponding Author: Dr. Vinnakota Sindhura, Flat No. 102, ELRS Residency, Krishna Nagar, Maharani Peta, Visakhapatnam-530002, Andhra Pradesh, India. E-mail: drsindhura.vinnakota@gmail.com

DOI: $10.14260 /$ jemds/2019/587

Financial or Other Competing Interests: None.

How to Cite This Article:

Prasad VS, Sindhura V, Kumar MN. Effectiveness of waist circumference in assessing the health risk by evaluating the cardio metabolic risk factors. J. Evolution Med. Dent. Sci. 2019;8(35):2703-2707, DOI: 10.14260/jemds/2019/587

Submission 08-07-2019,

Peer Review 18-08-2019,

Acceptance 24-08-2019,

Published 02-09-2019. 


\section{BACKGROUND}

Obesity is an important risk factor for cardio metabolic diseases like Diabetes, Hypertension, Dyslipidemia and Coronary Heart Disease (CHD). Several organizations like WHO, National Institutes of Health tried to classify Obesity on basis of weight using BMI.1,2 Several epidemiological studies revealed direct correlation between BMI and the risk of medical complications and mortality rate.3,4 Body fat distribution is also an important risk factor for obesity-related diseases. Excess abdominal fat also known as central or upper body fat is associated with increased risk of cardio metabolic disease. Janssen et $\mathrm{al}^{5}$ reported that increased visceral fat, as measured by MRI correlated well with Waist circumference (WC) than with body mass index (BMI). Zhu et $\mathrm{al}^{6}$ using the third National Health and Nutrition Examination Survey data reported that WC is more closely linked to cardiovascular disease risk factors than BMI. MRI can measure precisely the abdominal fat, but it is costlier. Hence Waist circumference is often used as a surrogate marker of abdominal fat mass as WC correlates with abdominal fat mass. ${ }^{7}$ Waist Circumference is associated well with cardio metabolic disease. ${ }^{8}$ The results presented by Kahn and Valdez reported that WC measurement correlated with elevated fasting triglyceride concentrations and accelerated mortality and served to validate the potential usefulness of WC in physical examination for clinical purposes. ${ }^{9}$ In developing countries like India, to address the consequences of epidemic of Diabetes and cardio vascular problems, WC measurement would offer useful, inexpensive tool for early screening and intervention. Jack Wang stressed Waist circumference, a simple, inexpensive and reliable tool that should be included as part of physical examinations in the doctor's office. ${ }^{10}$ Measuring WC can be useful in monitoring a patient's response to diet and exercise treatment because regular aerobic exercise can cause a reduction in both WC and cardio metabolic risk, without a change in BMI. ${ }^{11}$ Samuel Klein et al opined that further studies are needed to establish WC cut points that can assess cardio metabolic risk, not adequately captured by BMI and routine clinical assessments. ${ }^{12}$ Hence the present study aimed to evaluate the effectiveness of WC in assessing the Health risk by evaluating the cardio metabolic risk factors and also to validate the results of the previous studies.

\section{METHODS}

The sample included 238 middle aged, male factory employees randomly selected from 626 employees of a factory. Sample calculated by formula and as uploaded in Survey Software with 95\% confidence level. Blood samples are collected in fasting for Triglycerides, $\mathrm{LDL}, \mathrm{HDL}$ and $\mathrm{HbA}_{1} \mathrm{c}$ estimation, $\mathrm{BP}$ measurement and Waist measurement.

\section{Duration and Place of the Study}

238 middle aged, male factory employees randomly selected from a factory and consent was taken for the present study to utilize the data for analysis and presentation. The study spread for 10 months from the month of July, 2018 to the month of April, 2019. Ethical committee approval obtained. The variable Waist Circumference is taken as independent variable and
Triglycerides, LDL, HDL, HbA1c, BP are measured as independent variables. The confounding variables age, sex are under control by including male, middle aged, healthy subjects in the study. Female subjects and refusal to give informed consent are excluded.

\section{Waist Circumference}

Waist measurement taken midway between iliac crest and the lower edge of the rib cage. Basing on Waist Measurement, all the 238 subjects are categorized into 3 groups. 110 subjects in $\leq 90 \mathrm{cms}, 82$ subjects in $91 \mathrm{cms}-100 \mathrm{cms}, 46$ subjects in $>100$ cms.

\section{Triglyceride Levels}

All subjects are categorized into 3 groups, $\leq 150 \mathrm{mg} \%$, 151$399 \mathrm{mg} \%, \geq 400 \mathrm{mg} \%$

\section{LDL Levels}

All subjects are categorized into 3 groups, $\leq 100 \mathrm{mg} \%$, 101$129 \mathrm{mg} \%, \geq 130 \mathrm{mg} \%$

\section{HDL Levels}

All subjects are categorized into 3 groups, $\leq 40 \mathrm{mg} \%$, 41- 60 $\mathrm{mg} \%,>60 \mathrm{mg} \%$

\section{HbA1c Levels}

All subjects are categorized into 3 groups, $<5.7 \%, \geq 5.7-6.5 \%$, $\geq 6.5 \%$.

\section{BP Levels}

BP measured and all subjects are categorized into 4 groups, Normal: Systolic $\leq 120 \mathrm{~mm} \mathrm{Hg}$ and Diastolic $\leq 80 \mathrm{~mm}$ Hg. PreHypertension: Systolic 120-129 mm Hg and Diastolic $\leq 80 \mathrm{~mm}$ Hg. Stage 1 Hypertension: Systolic 130-139 mm $\mathrm{Hg}$ and Diastolic 80-89 mm Hg. Stage 2 Hypertension: Systolic $\geq 140$ $\mathrm{mm} \mathrm{Hg}$ and Diastolic $\geq 90 \mathrm{~mm} \mathrm{Hg}$.

\section{Statistical Techniques}

Chi-square is applied to test the goodness of fit to verify the distribution of observed data with assumed theoretical and expected frequencies. A null hypothesis is established. The difference between observed value and expected value calculated. (O-E). Square of the deviation calculated $(\mathrm{O}-\mathrm{E})^{2}$. (O$\mathrm{E})^{2}$ is divided by its expected frequency. (O-E $)^{2 / E}$. All the values obtained in step 4 are summed. $\Sigma\left[(\mathrm{O}-\mathrm{E})^{2 / E}\right]$. Degrees of freedom is calculated by formula $(\mathrm{C}-1)(\mathrm{R}-1)$. The table value of $\mathrm{X}^{2}$ found at the level of significance 0.05 and degrees of freedom. The table value is compared to the calculated value. If calculated value is more than the table value, the null hypothesis is rejected. If calculated value is less than the table value, the null hypothesis is retained.

\section{RESULTS}

Regarding Triglycerides concentrations, 238 subjects are distributed as follows. $53(48.2 \%)$ in $\leq 150 \mathrm{mg} \%, 33$ (30\%) in $151-400 \mathrm{mg} \%, 24(21.8 \%)$ in $>400 \mathrm{mg} \%$ in $\leq 90 \mathrm{cms}$ Waist circumference group, $16(19.5 \%)$ in $\leq 150 \mathrm{mg} \%, 36(43.9 \%)$ in $151-400 \mathrm{mg} \%, 30(36.6 \%)$ in $>400 \mathrm{mg} \%$ in the $91-100 \mathrm{cms}$ Waist Circumference group, 8 (17.4\%) in $\leq 150 \mathrm{mg} \%, 16$ 
(34.8\%) in $151-400 \mathrm{mg} \%, 22(47.8 \%)$ in $>400 \mathrm{mg} \%$ in the group $>100 \mathrm{cms}$ Waist circumference group. The percentile distribution of high Triglycerides is more in the group $>100$ cms Waist Circumference.

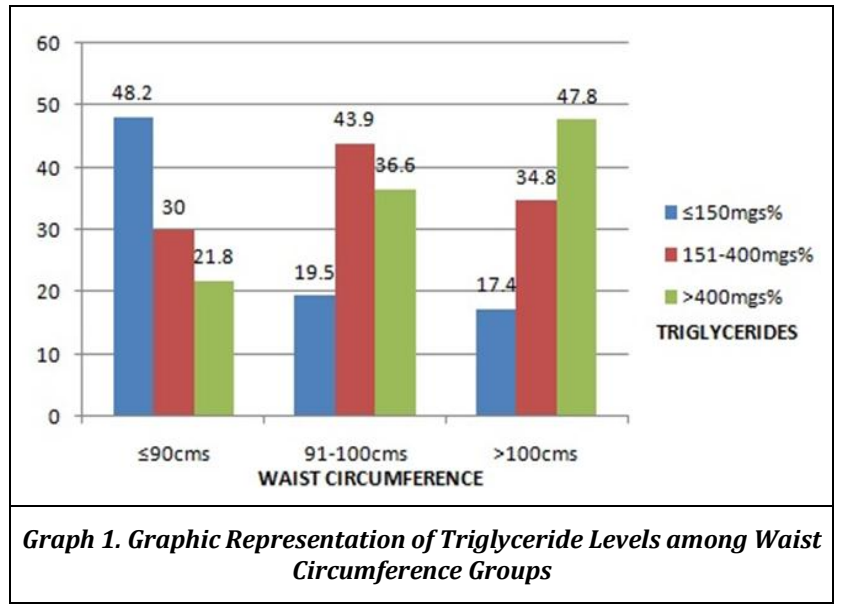

At Degrees of freedom, 4 and at significance of 0.05 , ChiSquare Table value is 9.488 . The calculated value is 26.16 which is more than Table value. So null hypothesis that there is no difference in Triglyceride levels among the various groups of Waist Circumference is rejected. Hence there is significant raise in the TG levels in the subjects with more waist circumference.

Regarding LDL concentrations, 238 subjects are distributed as follows. 52 (47.3\%) in $\leq 100 \mathrm{mg} \%, 34$ (30.9\%) in $101-130 \mathrm{mg} \%, 24(21.8 \%)$ in $>130 \mathrm{mg} \%$ in $\leq 90 \mathrm{cms}$ Waist circumference group, $16(19.5 \%)$ in $\leq 100 \mathrm{mg} \%$, 32 (39\%) in $101-130 \mathrm{mg} \%, 34$ (41.5\%) in $>130 \mathrm{mg} \%$ in the group $91-100$ cms Waist Circumference group, 10 (21.7\%) in $\leq 100 \mathrm{mg} \%, 13$ $(28.3 \%)$ in $101-130 \mathrm{mg} \%, 23(50 \%)$ in $>130 \mathrm{mg} \%$ in the group $>100 \mathrm{cms}$ Waist circumference group. The percentile distribution of high LDL is more in the group $>100 \mathrm{cms}$ Waist Circumference.

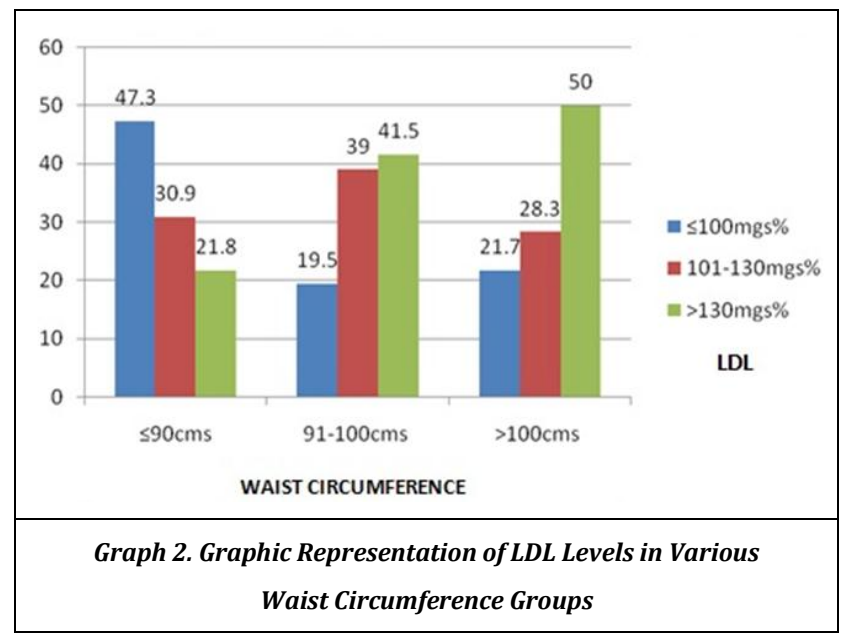

At Degrees of freedom, 4 and at significance of 0.05, ChiSquare Table value is 9.488. The calculated value is 24.11 which is more than table value. So, the null hypothesis that there is no difference in LDL levels among the various groups of Waist circumference is rejected. Hence there is significant raise in the LDL levels in the subjects with more waist circumference.
Regarding HDL concentrations, 238 subjects are distributed as follows. $58(52.7 \%)$ in $>60 \mathrm{mg} \%, 31(28.2 \%)$ in $41-60 \%, 21(19.1 \%)$ in $\leq 40 \mathrm{mg} \%$ in $\leq 90 \mathrm{cms}$ Waist circumference group, 17 (20.6\%) >60 mg\%, 30 (36.3\%) in 41$60 \mathrm{mg} \%$, 35 (42.4\%) in $\leq 40 \mathrm{mg} \%$ in the group $91-100 \mathrm{cms}$ Waist Circumference group, $7(15.2 \%)$ in $>60 \mathrm{mg} \%, 18$ $(39.1 \%)$ in $41-60 \mathrm{mg} \%, 21(45.7 \%)$ in $\leq 40 \mathrm{mg} \%$ in the group $>100 \mathrm{cms}$ Waist circumference group. The percentile distribution of low HDL is more in the group $>100 \mathrm{cms}$ Waist Circumference.

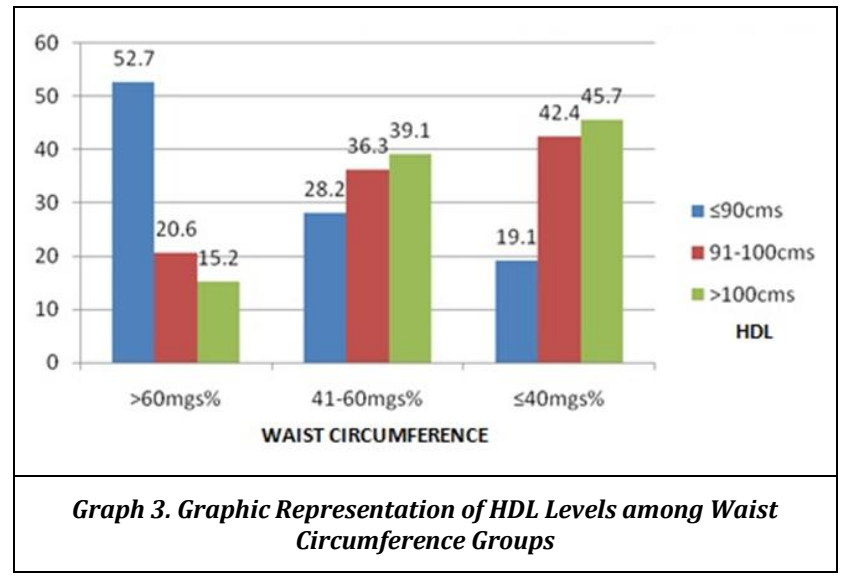

At Degrees of freedom, 4 and at significance of 0.05 , ChiSquare Table value is 9.488 . The calculated value is 32.89 which is more than table value. Hence the null hypothesis, that there is no difference in HDL levels among the various groups of Waist circumference is rejected. Hence there is significant raise in the HDL levels in the subjects with less waist circumference.

Regarding HbA1c levels, 238 subjects are distributed as follows. $86(78.2 \%)$ in $<5.7 \%, 14(12.7 \%)$ in $5.7-6.5 \%, 10$ $(9.1 \%)$ in $>6.5 \%$ in $\leq 90 \mathrm{cms}$ Waist circumference group, 56 $(68.3 \%)$ in $<5.7 \%, 14(17.1 \%)$ in $5.7-6.5 \%, 12(14.6 \%)$ in $>6.5 \%$ in $91-100 \mathrm{cms}$ Waist Circumference group, 7 (15.2\%) in $<5.7 \%, 19(41.3 \%)$ in $5.7-6.5 \%, 20(43.5 \%)$ in $>6.5 \%$ in the group $>100 \mathrm{cms}$ Waist circumference group. The percentile distribution of high HbA1c levels more in the group $>100 \mathrm{cms}$ Waist Circumference.

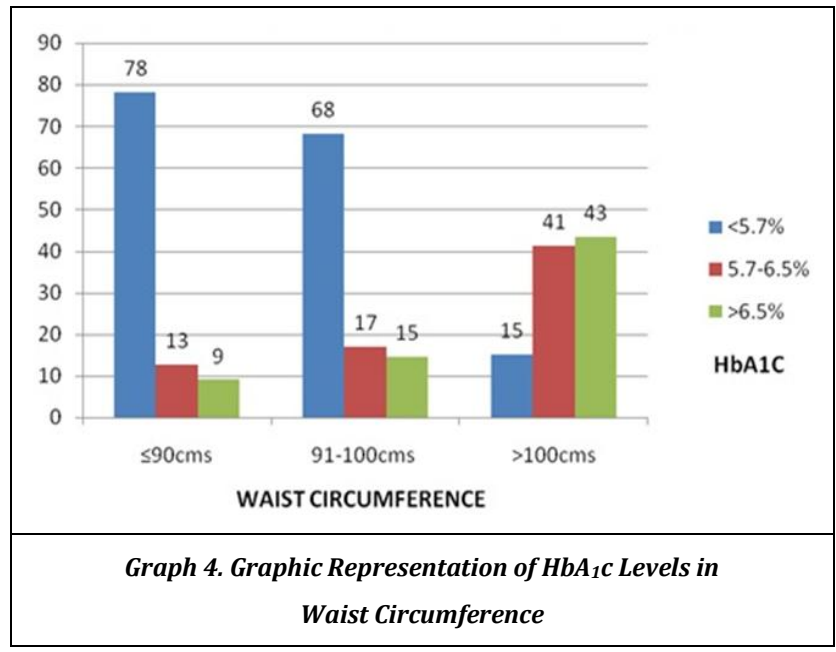

At Degrees of freedom, 4 and at significance of 0.05, ChiSquare Table value is 9.488 . The calculated value is 57.43 which is more than table value. Hence the null hypothesis that 
there is no difference in HbA1c levels among the various groups of waist circumference is rejected. Hence there is significant rise in the HbA1c levels in the subjects with more waist circumference.

Regarding BP levels, 238 subjects are distributed as follows. 88 (80\%) in Normal, 12 (10.9\%) in Pre Hypertension, $8(7.3 \%)$ in Stage 1 Hypertension, $2(1.8 \%)$ in Stage 2 Hypertension in $\leq 90 \mathrm{cms}$ Waist circumference group, 20 (24.4\%) in Normal, 19 (23.2\%) in Pre, 23 (28\%) in Stage 1, 20 (24.4\%) in Stage 2 in 91-100 cms Waist Circumference group, $5(10.9 \%)$ in Normal, 18 (39.1\%) in Pre, 11(23.9\%) in Stage 1, $12(26.1 \%)$ in Stage 2 in the group $>100 \mathrm{cms}$ Waist circumference group. The percentile distribution of Normal BP is more in the group $\leq 90 \mathrm{cms}$ Waist Circumference.

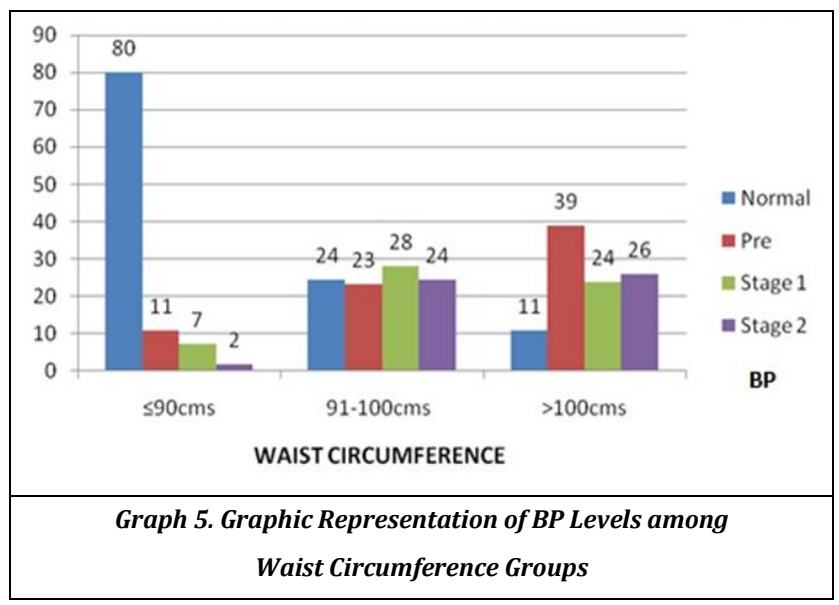

At degrees of freedom 6 and at the significance level of 0.05 , the Table value is 12.592 . The calculated value is 94.72 which is more than the table value, hence the null hypothesis that there is no difference in Blood Pressure levels among the various groups is rejected. There is significant rise in the Blood pressure levels in the subjects with more waist circumference. The percentage of normal BP is more in Low Waist circumference groups.

\section{DISCUSSION}

\section{Triglycerides and Waist Circumference}

The Null hypothesis that there is no difference in Triglyceride levels among the various groups of Waist Circumference is tested with Chi-square. With Df 4 and at 0.05 significant levels, Chi-Square table value is 9.488 . The calculated value is 26.16 which is more than Table value. So null hypothesis that there is no difference in Triglyceride levels among the various groups of Waist Circumference is rejected. $47.8 \%$ subjects of more than $100 \mathrm{cms}$ waist circumference documented more than $400 \mathrm{mg} \%$ Triglyceride levels whereas only $21.8 \%$ had more than $400 \mathrm{mg} \%$ Tg levels in $\leq 90 \mathrm{cms}$ waist circumference group. Hence the study revealed that there is significant raise in the TG levels in the subjects with more waist circumference.

\section{Low Density Lipoproteins and Waist Circumference}

The Null hypothesis that there is no difference in Low Density Lipoproteins levels among the various groups of Waist Circumference is tested with Chi-square. With Df 4 and at 0.05 significant levels, Chi-Square table value is 9.488. The calculated value is 24.11 which is more than Table value. So null hypothesis that there is no difference in Low Density Lipoproteins levels among the various groups of Waist Circumference is rejected. $50 \%$ subjects of more than $100 \mathrm{cms}$ waist circumference documented more than $130 \mathrm{mg} \% \mathrm{LDL}$ levels whereas only $21.8 \%$ had more than $130 \mathrm{mg} \%$ LDL levels in $\leq 90 \mathrm{cms}$ waist circumference group. The study revealed that there is significant raise in the LDL levels in the subjects with more waist circumference.

\section{High Density Lipoproteins and Waist Circumference}

The Null hypothesis that there is no difference in High Density Lipoproteins levels among various groups of Waist Circumference is tested with Chi-square. With Df 4 and at 0.05 significant levels, Chi-Square table value is 9.488. The calculated value is 32.89 which is more than Table value. So null hypothesis that there is no difference in High Density Lipoproteins levels among the various groups of Waist Circumference is rejected. $45.7 \%$ subjects of more than 100 cms waist circumference documented less than $40 \mathrm{mg} \% \mathrm{HDL}$ levels whereas only $19.1 \%$ only documented less than $40 \mathrm{mg} \%$ HDL levels in $\leq 90 \mathrm{cms}$ waist circumference group. The study revealed that there is significant diminution in the HDL levels in the subjects with more waist circumference.

\section{HbA1c Levels and Waist Circumference}

The Null hypothesis that there is no difference in Glycosylated Haemoglobin (HbA1c) levels among various groups of Waist Circumference is tested with Chi-square. With Df 4 and at 0.05 significant levels, Chi-Square table value is 9.488. The calculated value is $\mathbf{5 7 . 4 3}$ which is more than Table value. So null hypothesis that there is no difference in HbA1c levels among the various groups of Waist Circumference is rejected. $43.5 \%$ subjects of more than $100 \mathrm{cms}$ waist circumference documented more than $6.5 \%$ HbA1c levels whereas only $9.1 \%$ subjects documented more than $6.5 \%$ HbA1c levels in $\leq 90 \mathrm{cms}$ waist circumference group. The study revealed that there is significant increase in the HbA1c levels in the subjects with more waist circumference.

\section{Blood Pressure Levels and Waist Circumference}

The Null hypothesis that there is no difference in Blood Pressure levels among various groups of Waist Circumference is tested with Chi-square. With Df 6 and at 0.05 significant levels, Chi-Square table value is 12.592 . The calculated value is 94.72 which is more than Table value. So null hypothesis that there is no difference in BP levels among the various groups of Waist Circumference is rejected. The study revealed that there is significant increase in the Blood Pressure levels in the subjects with more waist circumference. Only $10 \%$ subjects had normal BP in the group of more than $100 \mathrm{cms}$ waist circumference whereas $80 \%$ of cases of normal BP (80\%) found in the low Waist Circumference group ( $<90 \mathrm{cms})$.

\section{CONCLUSIONS}

Waist Circumference is well correlated with Triglycerides, LDL, HbA1c and BP readings but inversely correlated with HDL. The high levels of Waist Circumference correlated well with several biomarkers of Cardiovascular Disease and Diabetes. The present study correlated well with the previous 
studies of waist circumference and cardio metabolic risk factors. The present study emphasizes the importance of Waist circumference which is cheap, simple and reliable tool not only for screening but also for monitoring to assess the diet and exercise regimen. Every doctor should include the Waist Circumference as part of physical examination to address the epidemic of Obesity and Diabetes.

\section{REFERENCES}

[1] World Health Organization. Obesity: preventing and managing the global epidemic: Report of a WHO Consultation on Obesity. Geneva, World Health Organization, 1998: p. 7-9.

[2] National Institutes of Health. National Heart, Lung and Blood Institute: Clinical guidelines on the identification, evaluation and treatment of overweight and obesity in adults - the evidence report. Obes Res 1998;6(Suppl 2):51S-209S.

[3] Colditz GA, Willett WC, Rotnizky A, et al. Weight gain as a risk factor for clinical diabetes mellitus in women. Ann Intern Med 1995;122(7):481-6.

[4] Calle EE, Thun MJ, Petrellin JM, et al. Body-mass index and mortality in a prospective cohort of U.S. adults. N Engl J Med 1999;341(15):1097-105.

[5] Janssen I, Heymsfield SB, Allison DB, et al. Body mass index and waist circumference independently contribute to the prediction of non-abdominal, abdominal subcutaneous and visceral fat. Am J Clin Nutr 2002;75(4):683-8.
[6] Zhu S, Wang Z, Heshka S, et al. Waist circumference and obesity-associated risk factors among whites in the third National Health and Nutrition Examination Survey: clinical action thresholds. Am J Clin Nutr 2002;76(4):7439.

[7] Pouliot MC, Despres JP, Lemieux S, et al. Waist circumference and abdominal sagittal diameter: best simple anthropometric indices of abdominal visceral adipose tissue accumulation and related cardiovascular risk in men and women. Am J Cardiol 1994;73(7):460-8.

[8] Kissebah AH, Vydelingum N, Murry R, et al. Relation of body fat distribution to metabolic complications of obesity. J Clin Endocrinol Metab 1982;54(2):254-60.

[9] Kahn HS, Valdez R. Metabolic risks identified by the combination of enlarged waist and elevated triacylglycerol concentration. Am J Clin Nutr 2003;78(5):928-34.

[10] Wang J. Waist circumference: a simple, inexpensive and reliable tool that should be included as part of physical examinations in the doctor's office. Am J Clin Nutr 2003;78(5):902-3.

[11] Dekker MJ, Lee S, Hudson R, et al. An exercise intervention without weight loss decreases circulating interleukin- 6 in lean and obese men with and without type 2 diabetes mellitus. Metabolism 2007;56(3):332-8.

[12] Klein S, Allison DB, Heymsfield SB, et al. Waist circumference and cardiometabolic risk: a consensus statement from shaping America's health: Association for Weight Management and Obesity Prevention, NAASO, the Obesity Society, the American Society for Nutrition, and the American Diabetes Association. Diabetes Care 2007;30(6):1647-52. 